\title{
AUTOMATED QUALITY ASSESSMENT OF CONSTRUCTED SURFACES THROUGH 'INTELLIGENT' IMAGE PROCESSING
}

\author{
By Yassir AbdelRazig ${ }^{1}$, Luh-Maan Chang ${ }^{2}$, and Miroslaw Skibniewski ${ }^{3}$ \\ 'Ph.D., Div. of Constr. Engrg. and Mgmt.. School of Civ. Engrg.. \\ Purdue Univ.. West Lafayette, IN 47907-1294.USA. E-mail: abdelraz@ecn.purdue.edu \\ ${ }^{2}$ Assoc. Prof.. Div. of Constr. Engrg. and Mgmt.. School of Civ. Engrg.. \\ Purdue Univ., West Lafayette, IN 47907-1294. USA. \\ ${ }^{3}$ Prof., Div. of Constr. Engrg. and Mgmt.. School of Civ. Engrg.. \\ Purdue Univ., West Lafayette, IN 47907-1294. USA.
}

\begin{abstract}
Computerized intelligent systems can simulate human expertise, as well as analyze and process vast amounts of data instantly. This paper presents a hybrid intelligent computerized model for constructed surfaces quality assessment. The assessment of steel bridge coating is used to exemplify the model. The model will automate the assessment process by using computers to analyze digital images of the areas to be assessed in order to identify and measure defect patterns. Most techniques, currently used in construction and infrastructure assessment and quality inspection, rely merely on subjective criteria. Such subjective, and hence inconsistent, assessment techniques have been identified as a critical obstacle to effective infrastructure or constructed facilities management.
\end{abstract}

Keywords: Quality assessment, image processing, pattern recognition, neural networks.

\section{INTRODUCTION}

The Federal Highway Administration estimates that $45 \%$ of the approximately $1 / 2$ million bridges in the U.S. are deficient. These deficiencies have led to a $\$ 90$ billion of needed repair and maintenance [16]. In spite of the money spent, the effort to fix those deficiencies is progressing slowly, particularly in the area of coating quality assessment, a crucial element of steel bridge quality [9].

Moreover, the currently used assessment techniques are subjective, time consuming, and rely mostly on human visual inspection. These techniques have not taken advantage of advanced technologies, especially computer technology [10].

Such subjective assessment methods have been identified as a critical obstacle to effective infrastructure management [2]. Therefore, more objective. accurate, and reliable assessment techniques need to be explored to improve the quality of infrastructure and constructed facilities [3].

This paper presents a computer-based decision support model for image data analysis in order to improve the construction quality assessment process. This paper will first present a brief theoretical background for image processing. pattern recognition, and neural networks. Then, the architecture of the computerized decision support model will be described. Finally, an example of an image with rust areas will be used to exemplify the concepts of the decision support system and its application. Finally, conclusions and application framework will be presented.

\section{BACKGROUND}

\section{I Image Processing}

Generally, an image is formed by means of visible light. The light emanates from a light source and interacts with the surfaces of objects. After that, part of the light is captured by an image formation system. like a camera, which produces a twodimensional distribution of the light defined on the image plane. It is this distribution of light that we call an image [14].

The recognition of an object in an image is a complex process that involves a wide range of elaborate techniques. The first step in a typical image processing application is image acquisition, which is acquiring an image in digital format. The term "image" refers to a two-dimensional light intensity function $f(x, y)$ where $x$ and $y$ are geometrical coordinates and the value of $f$ at any point $(x, y)$ is 
proportional to the brightness or gray level of the image at that point [14].

A digital image can be considered as a matrix in which each row and column represents a point in the image and the corresponding matrix element value represents the gray level at that point. The elements of such an array are called picture elements or pixels.

After a digital image has been acquired, the next step is preprocessing, which corresponds to low-level processing. A number of prepossessing techniques is available in practice [6]. These may include gray scale manipulation, noise filtering, isolation of regions, geometric correction. and restoration.

The next stage is the recognition stage, which corresponds to high-level processing and it deals with classification. It assigns a classification to an object based on the parameters provided by its descriptors. Conventional classification techniques are grouped into two categories: supervised and unsupervised techniques. In a supervised mode, classifiers learn with the help of training sets. In the case of unsupervised mode, classifiers learn without training sets. Neural networks represent a powerful alternative to conventional classifiers [12].

\subsection{Statistical Pattern Recognition}

Pattern recognition techniques assign a physical object or an event to one of several pre-specified categories or classes [4]. Thus, a pattern recognition system can be viewed as an automatic decision rule; it transforms measurements on a pattern into class assignments. There are many algorithms for statistical pattern recognition. One of the most widely used algorithms is the K-Means algorithm [13].

The K-Means Algorithm self-organizes data to create clusters. This algorithm fits the coating inspection application well because of the number of clusters need to be predetermined, which is the case in the inspection process, where two groups are identified: defects and non-defects [7].

The algorithm uses a sample of feature vectors $V$ $=\left\{x_{(I)}, \ldots, x_{(Y)}\right\}$ from a population $P$, but requires the number $K$ of clusters to be given, $K<Y$. The process begins by assigning the first $K$ sample feature vectors $x_{(1)}, \ldots, x_{(Y)}$ to be centers $z_{(1)}, \ldots, z_{(K)}$. respectively, for the $K$ clusters. The algorithm then assigns each of the remaining $Y$-K sample feature vectors $x_{(K+1)}, \ldots, x_{(Y)}$ to the cluster whose center is closest. Then, the sample feature vectors for each $K^{\text {th }}$ cluster, in turn, are averaged to determine a new cluster center $z_{(K)}$ for each $K^{\text {th }}$ cluster. Next, each of the $Y$ sample vectors is again assigned to the class to whose new representative center it is closest [15].

This process of generating new centers by averaging each cluster and then reassigning all vectors by minimum distance to the new centers is repeated until no clusters change further, in which case the algorithm is finished [13].

\subsection{Artificial Neural Nenwork}

Neural network technology is an accepted widely used field of artificial intelligence. Neural networks have biological basis as their design and functionality emulates the human brain.

Tsoukalas and Uhrig [18] defined an artificial neural network as: "A data processing system consisting of a large number of simple, highly interconnected processing elements (artificial neurons) in architecture inspired by the structure of the cerebral cortex of the brain."

Generally, neural networks are trained so that a particular input leads to a specific target output. Figure 1 illustrates the general functionality architecture for training. The network is adjusted, based on a comparison of the output and the target. until the output matches the target.

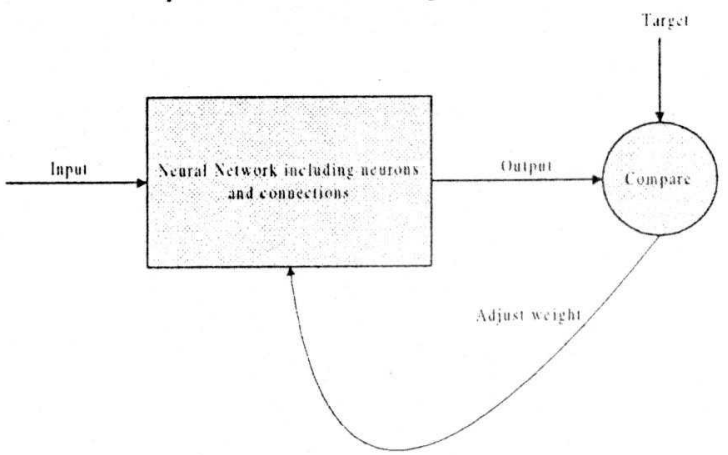

Figure 1. Neural Network Functionality

A popular artificial neural network model is the back propagàtion network model. The basic back propagation model is a three-layered forward architecture. The first layer is the input layer, the second layer the hidden layer, and the third layer is the output layer. Each layer contains a group of nodes that are linked together with nodes from other layers by connections among the nodes. Layers are connected only to the adjacent layers.

The network is a feed-forward network, which means a unit's output can only originate from a lower level, and a unit's output can only be passed to a higher level. Figure 2 illustrates the basic architecture of a feed forward neural network [8]. 


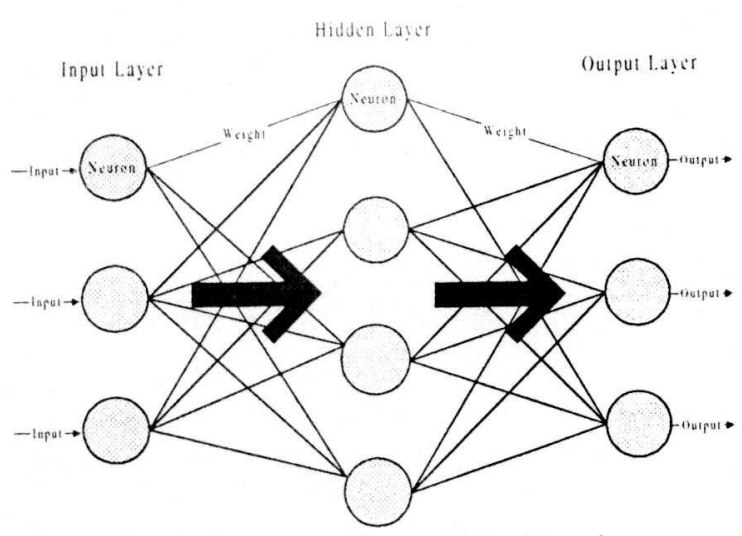

Figure 2. Neural Network Architecture

The input layer receives the features of the data that are entered into the neural network. If $n$ feature values are to be entered into the input layer, then there must be $n$ nodes, where $n$ is the number of features supplied to the net. A single feature value is inputted into a single input node [19].

\section{THE DECISION SUPPORT MODEL}

The basic concept of the decision support model is to acquire digital images of objects to be assessed and identify defects using image analysis techniques. Moreover, sample images will be used to train the system to acquire expert knowledge in identifying the defects and using this knowledge to later assess other images [1]. Figure 3 illustrates the model architecture. It consists of four main stages connected sequentially [11].

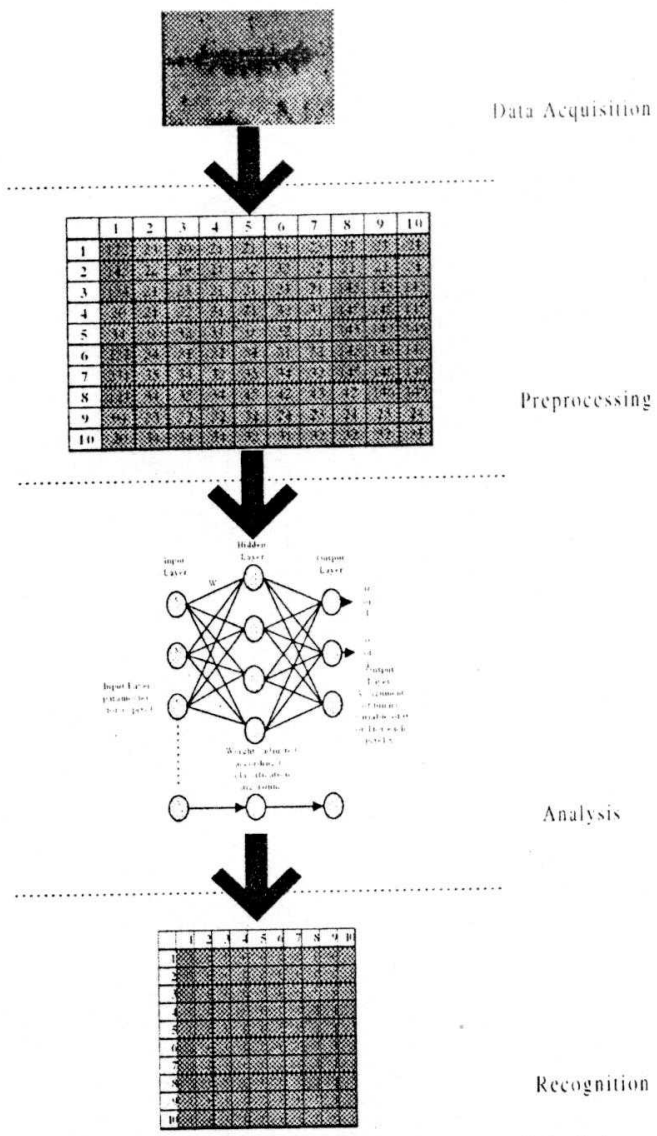

Figure 3. The Hybrid System Model

The first stage is Data Acquisition, where digital images of the steel bridge coating (objects) are obtained using a digital camera. After acquisition. the images are transferred to the computer on site or to a remote office via any communication protocol. This stage is illustrated in Figure 4.

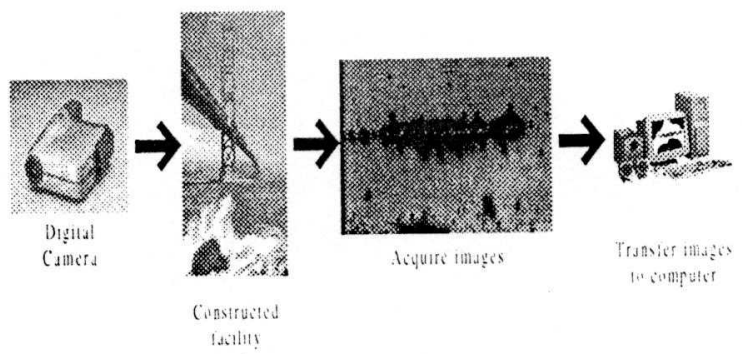

Figure 4. Data Acquisition Phase

The next stage is the Preprocessing stage. where image analysis techniques are used to analyze and enhance the image by applying algorithms such as filtering and edge detection. Moreover, image preprocessing is used to obtain the numerical parameters of the image such as the gray level and brightness in a numerical format. Figure 5 illustrates the pre-processing stage. 


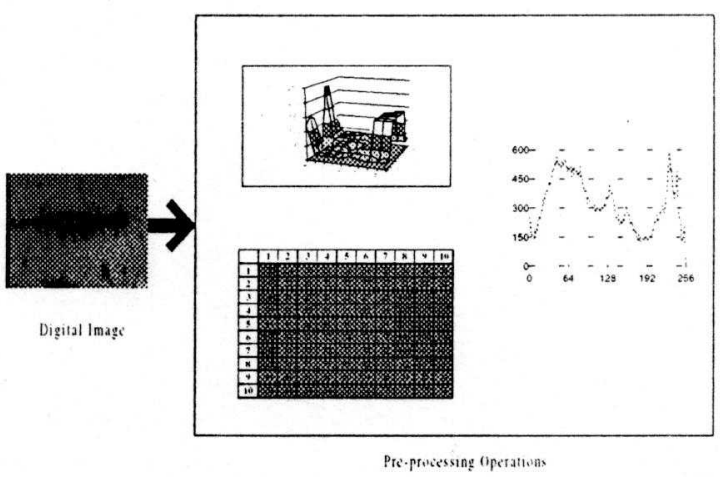

Figure 5. Pre-processing Phase

The next stage is the Analysis stage. After being trained, neural networks are used to identify defects in the images by assigning binary variable of 0 or 1 for each pixel in the image. During the network training, the neural network is fed with different images and their parameters such as the pixels' gray levels. The network is also fed mapped values of 0 or 1 for each pixel value. The network will learn to assign the binary variable 0 or 1 for different scenarios according to image parameters. Figure 6 illustrates the Analysis phase.

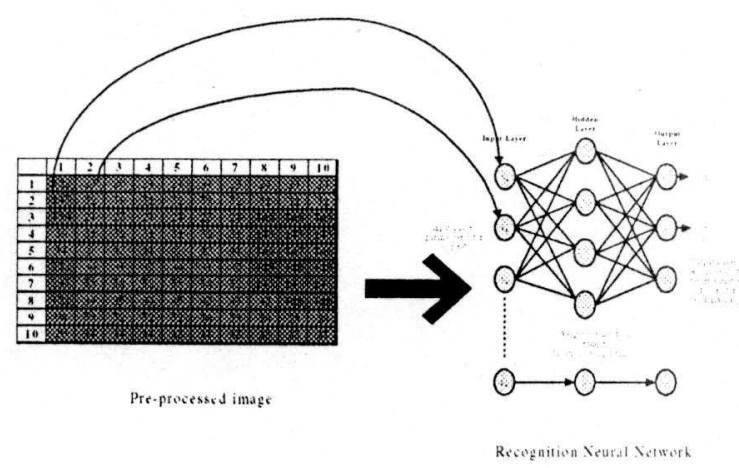

Figure 6. Analysis Phase

The final stage is the Recognition stage, where quantitative measures of defects are obtained from the output of the previous stage. From mapped output of the neural network, the whole image is represented as 0 's or 1 's. The 1 values represent the defect; hence, defects can be identified and quantitatively measured. The system can be trained to identify different types of defects according to the specific application. The Recognition stage is shown in Figure 7.

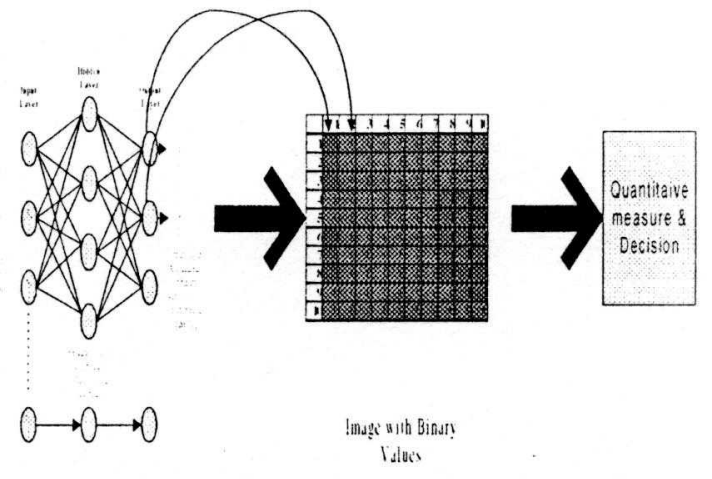

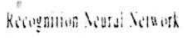

Figure 7. Assessment Results Phase

\section{EXAMPLE}

The following example illustrates how a simple (5 pixel $x 5$ pixel) image could be used to illustrate the methodology of the hybrid model [1].

\section{Step 1 Data Acquisition}

A digital image of a steel bridge coating area is obtained and transferred to the computer.

\section{Step 2 Preprocessing}

Image analysis software is used to enhance the image and to represent the image in numerical format. Figure 8 shows the sample image with the gray level shown for each pixel.

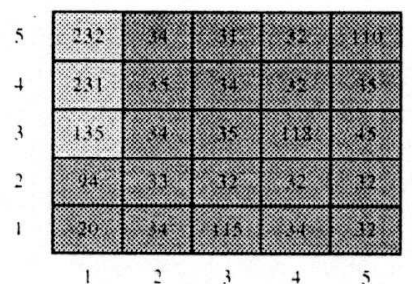

Figure 8. Example Image Gray Levels

The next step is to apply the statistical pattern recognition algorithm to classify the numerical values into two clusters: rust or no rust. The result of this classification is then passed to the third step. Given the data set obtained from the image numerical presentation in Figure 8, the K-means Algorithm is applied.

$V=\{2,32,34,31,32,110,231,35,34,32,35,135$, $34,35,118,45,94,33,32,32,32,20,34,115,34$. 32\}.

Select randomly, the first two samples as initial centers.

$\mathrm{z}_{1}=2.32 \quad \mathrm{z}_{2}=34$

Next assign each sample to the class with the closest center, i.e. to class $I$ if it is closer to $z_{1}=2.32$ and to class 2 if it is closer to $z_{2}=34$. 
The following table shows the difference between each pixel value and the initial two centers and hence to which class it is assigned. Note that the difference is calculated based on the gray level, not based on the location of the pixel. The gray level is considered a third dimension for each pixel.

\begin{tabular}{|c|c|c|c|c|}
\hline P'ixel Value & $\begin{array}{l}\text { Dillerence finom } Z_{1} \\
Z_{1}=231\end{array}$ & $\begin{array}{c}\text { Diflerence in } Z_{2} \\
Z Z=3.4\end{array}$ & Claw 1 & Class 2 \\
\hline 232 & 0 & 198 & $x$ & \\
\hline kil. & 11. & 109 & 4 & \\
\hline 135 & 97 & 101 & $x$ & \\
\hline \% & $x \times$ & $6 \%$ & : & x. \\
\hline 20 & 212 & 14 & & $\mathrm{x}$ \\
\hline 4. & 14 & : & ;. & *) \\
\hline 35 & 197 & 1 & & $\mathrm{x}$ \\
\hline x. & 12 & \%x. & (2) & $x$ \\
\hline 33 & 199 & 1 & & $x$ \\
\hline . & 188 & 69 & & $x$ \\
\hline 31 & 201 & 3 & & $x$ \\
\hline 84 & 16 & 48 & \% & x \\
\hline 35 & 197 & 1 & & $x$ \\
\hline 32 & ihr. & 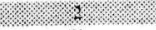 & ג & . \\
\hline 115 & 117 & 81 & & $x$ \\
\hline s/ & 3iv & : & . & $x$ \\
\hline 32 & 200 & 2 & & $x$ \\
\hline (x) & - & 6 & 1. & $x$ \\
\hline 32 & 200 & 2 & & $x$ \\
\hline x & W & m. & . & 8 \\
\hline 110 & 122 & 76 & & $x$ \\
\hline 凊. & xy' & ; & m. & $\mathrm{s}$ \\
\hline 45 & 187 & 11 & & $\mathrm{x}$ \\
\hline 12. & 24. & 2. & . & 8 \\
\hline 32 & 200 & 2 & & $\mathrm{x}$ \\
\hline
\end{tabular}

Table 1. First Iteration for K-means Algorithm

$C_{1}=\{232,231,135\}$.

$C_{2}=\{34,31,32,110,35,34,32,35,34,35,118$, $45,94,33,32,32,32,20,34,115,34,32\}$.

Figure 9 shows the initial clustering.

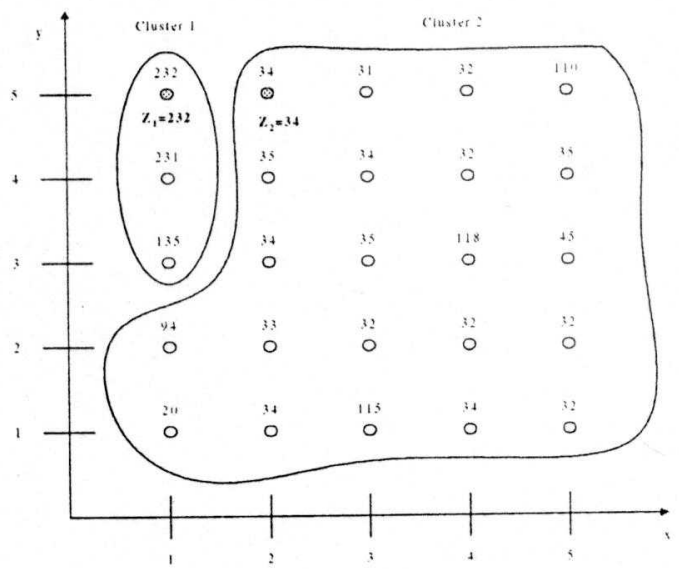

Figure 9. Initial Clusters

Next, each cluster's samples are averaged to obtain new optimal centers.

New center $z_{1}=\sum \frac{\text { Gray levels values in cluster I }}{\text { number of points in cluster } 1}$ $=199.3$

New center $z_{2}=\sum \frac{\text { Gray levels values in cluster } 2}{\text { number of points in cluster } 2}$ $=50.78$

The same procedure is applied again to find the distance between each sample and the two centers and assign the sample to the cluster with the closest center.

\begin{tabular}{|c|c|c|c|c|}
\hline P'Pixel Valux & $\begin{array}{c}\text { Dillerence trom } \gamma_{1} \\
\left.y_{1}=1 \%\right)_{3}\end{array}$ & 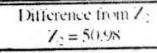 & Claw 1 & (7) \\
\hline 232 & 327 & 18122 & $x$ & \\
\hline 231 : & $41 \%$ & 18622 & $x$ & \\
\hline 135 & $6+3$ & $x+22$ & $x$ & \\
\hline 0 & $108 \%$ & 437 & (x) & $x$ \\
\hline 30 & 179.3 & 301.78 & & $s$ \\
\hline 4 & 1643. & $16 \%$ & i: & $x$ \\
\hline 35 & $10: 3$ & $15.7 x$ & & $x$ \\
\hline 4 & $10 \%$ & $16 \%$ & 2. & $x$ \\
\hline 3 & 166.3 & 17.78 & & $x$ \\
\hline 4 & $164 \%$ & $16 \%$ & min & 8 \\
\hline 31 & $16 \times 3$ & $19,7 x$ & & $\mathrm{x}$ \\
\hline 14 & $16 r 4 \%$ & 1678 & . & $x$ \\
\hline 35 & 1643 & 15.78 & & 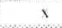 \\
\hline 12 & $10 \% 3$ & 1878 & $2:$ : & $x$ \\
\hline 115 & 4.3 & $64: 2$ & & $x$ \\
\hline $3 \%$ & 1673 & 1878 & ব & a \\
\hline 32 & 167.3 & 18.78 & & $x$ \\
\hline 118 & 818 & $6 \% 2$ & * & x. \\
\hline 32 & 167.3 & 18.78 & & $x$ \\
\hline 4 & $163 \%$ & 168 & . & $x$ \\
\hline 110 & 80.3 & 59.22 & & 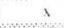 \\
\hline 35 & $14 \pi$ & 1878 & m. & $x$ \\
\hline 45 & 15.3 & $5.7 x$ & & $x$ \\
\hline $4 \%$ & 1678 & $18 \%$ & : & $\mathrm{x}$ \\
\hline 32 & 167.3 & 16.78 & & s \\
\hline
\end{tabular}

Table 2. Second Iteration for K-means Algorithm

$C_{1}=\{232,231,135\}$

$C_{2}=\{34,31,32,110,35,34,32,35,34,35,118$. $45,94,33,32,32,32,20,34,115,34,32\}$.

The class assignment did not change and hence the algorithm is finished. The final clusters are shown in Figure 10.

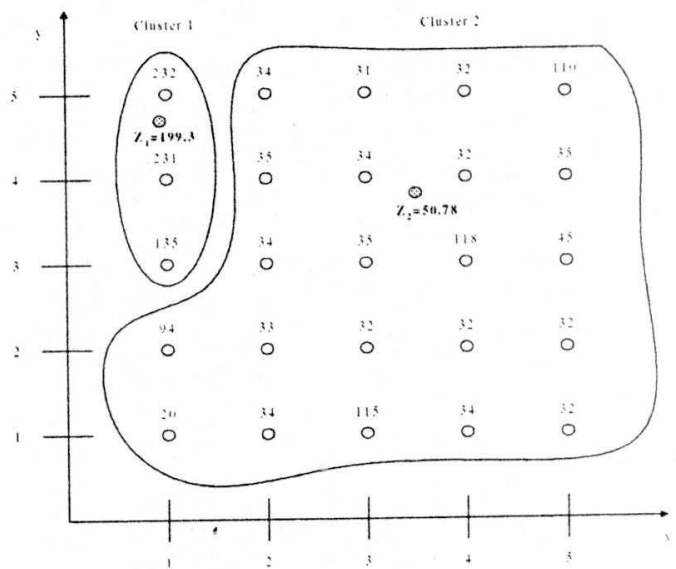

Figure 10. Final Clusters

Finally. each sample is assigned a binary variable of 1 if it belongs to cluster 1 and 0 if it belongs to cluster 2 . The image representation will be as shown in Figure 11.

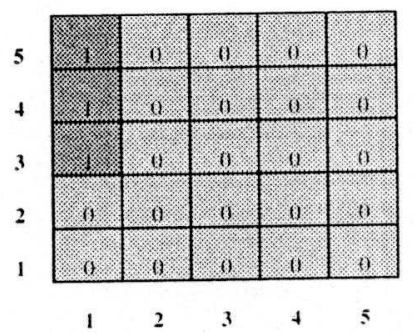


Figure 11. Rust Identification in the Image

Step 3 Analysis

Data from the image shown in Figures 8 and 11 will be used to train the neural network. The purpose of using the neural network is to accommodate for deficiencies in images' quality or the existence of noise in images [1].

The complete neural network will consist of three layers: the input layer, the hidden layer, and the output layer. The input layer consists of the image data and has four elements: The $\mathrm{x}$-axis location of the pixel, then the $y$-axis location of the pixel, the value of the gray level of the pixel, and finally the difference between the gray level of the pixel and a predetermined threshold value.

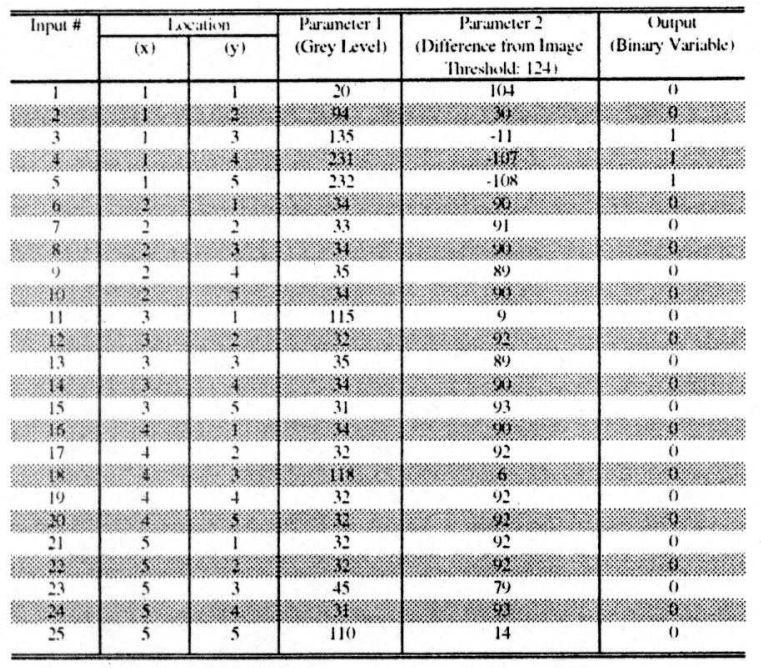

Table 3. Neural Network Data Input

Many actual images of sizes such as 600 pixels by 400 pixels will be used for the model implementation to obtain reliable training results. A statistical sampling plan will be adopted for image acquisition and assessment decisions [5].

\section{CONCLUSIONS AND FUTURE APPLICATION}

This paper presented a computerized decision support model for constructed surfaces quality assessment. The application of the model was illustrated using steel bridge coating assessment. The purpose of this research is to propose an approach that is objective, quantitative, and reliable.

This hybrid model applies concepts from the fields of, image processing, pattern recognition, and neural networks. The primary purpose of the research is to automate the assessment process by acquiring digital images of the areas to be assessed and analyzing the images using computers to identify and measure defects.
The system may overcome the subjectivity and inconsistency of human visual assessment by analyzing digital images with computers. Unlike the human eye, the computer can recognize and distinguish millions of colors and 256 shades of gray. By analyzing different parameters and characteristics for each pixel in any given image. the system can recognize defect patterns undetectable by humans. Moreover, the system can accurately measure the extent of defect.

It is hoped that this system can substantially improve the quality assessment process by making it more objective, quantitative, consistent, and accurate. However, in order to apply the system to practical quality assessment in the field, it is necessary to implement it as part of an integrated system utilizing light reflectance sensors and measurement units mounted on a mobile platform equipped with an articulated boom and a motion controller, thus constituting an "intelligent" robotic system [17].

Robotic technologies promise substantial returns on investment. However, there is a lack of objective evaluations of technology performance and consistent metrics for quantifying such returns, which should be undertaken as a follow up to technology development and pilot application.

\section{REFERENCES}

[1] AbdelRazig, Y. (1999). "Construction Quality Assessment: A Hybrid Decision Support Model Using Image Processing and Neural Learning for Intelligent Defects Recognition" Ph.D. thesis, Purdue University, West Lafayette, IN.

[2] Abraham, D., Iseley, T., Prasanth, R., and Wirahadikusumah, R. "Integrating Sensing Technologies for Underground Utilities Assessment." Infrastructure Condition Assessment: Art. Science. and Practice. Proceedings of the conference sponsored by the Facilities Management Committee of the Urban Transportation Division of the American Society of Civil Engineers, August 25-27. 1997. Boston, MA, 316-325.

[3] ASCE (1988). Manual of Professional Practice: Quality in the Constructed Project: A Guideline for Owners, Designers, and Constructors. American Society of Civil Engineers. 17-22.

[4] Bunke. H. (1992). Advances in Structural and Syntactic Pattern Recognition, World Scientific, River Edge, NJ.

[5] Chang, L. and Hsie, M. (1995). "Developing acceptance sampling methods for quality 
construction." Journal of Construction Engineering and management, 121(2), 246-253.

[6] Croall, I. F., and Mason, J. P. (1992). Industrial Applications of Neural Networks, Springer-Verlag New York Inc., New York, NY.

[7] De La Blanca, N. (1992). Pattern recognition and Image Analysis. World Scientific, River Edge, NJ.

[8] Haykin, S. (1999), Neural Networks: A Comprehensive Foundation, Prentice Hall. Upper Saddle River, NJ.

[9] Hsie, M. (1994). “Computer Aided Acceptance Planning: Generating Acceptance Parameters and Stratified Sampling Plans Through Neural Network Learning Ability and CAD Modeling." Ph.D. Thesis. Purdue University, West Lafayette, IN. 1-2.

[10] Hunt, V., Helmicki, A., and Aktan, E. (1997). "Instrumented monitoring and nondestructive evaluation of highway bridges." Infrastructure Condition Assessment: Art, Science, and Practice. Proceedings of the conference sponsored by the Facilities Management Committee of the Urban Transportation Division of the American Society of Civil Engineers, August 25-27, 1997, Boston, MA. 121-130.

[11] Kandel, A., and Langholz, G. (1992). Hybrid Architectures for Intelligent systems, CRC Press, Boca Raton, FL.

[12] Liu, J. (1997). "Quality prediction for concrete manufacturing." Automation in Construction. 5(6), 491-499.

[13] Ripley, B. D. (1996). Pattern Recognition and Neural Networks, Cambridge University Press, New York, NY.

[14] Russ, J.C. (1995). The Image Processing Handbook, CRC Press, Inc., Boca Raton, FL

[15] Schalkoff, R. (1992). Pattern Recognition: Statistical, Structural, and Neural Approaches, John Wiley \& Sons, Inc., New York, NY.

[16] Shubinsky, G. (1994). "Application of Optical Imaging Method for Bridge Maintenance and Inspection." ITI Technical Report No. 4, Northwestern University, Evanston, IL.

[17] Skibniewski, M. (1988): "Robotics in Civil Engineering," Computational Mechanics Publications and Van Nostrand Reinhold. Southampton, Boston, New York, ISBN 0-44231925-8. $233 \mathrm{pp}$.
[18] Tsoukalas. H., and Uhrig. E. (1997). Fuzal and Neural Approaches in Engineering. John Wiley \& Sons, Inc.. New York. NY.

[19] Yu, W. (1996). "A Neuro-Fuzzy KnowledgeBased Multi-Criterion Decision Model for Constructability Analysis and Improvement of Construction Technologies" Ph.D. thesis, Purdue University. West Lafayette, IN. 